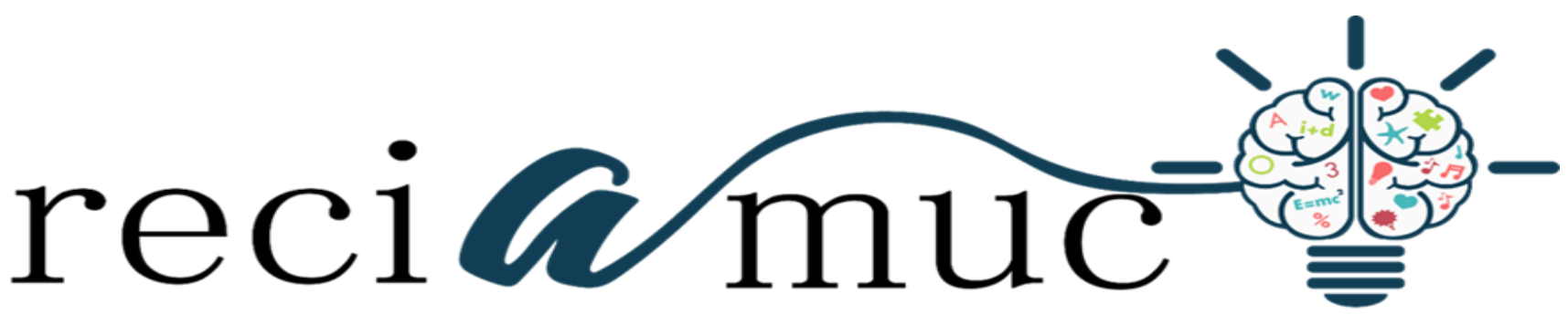

Revista cientifica de investigación actualización del mundo de las ciencias

Stefany Gianella Campuzano Lupera ${ }^{\text {a; }}$ Carlos Alexander Bajaña Gómez ${ }^{\text {b; Elvia }}$ María Córdova Cedeño ${ }^{c}$; Carlos Eduardo Baque Castro ${ }^{\mathrm{d}}$

VIH/SIDA: Pruebas y su Efectividad

HIV/AIDS: Testing and its Effectiveness

Revista Científica de Investigación actualización del mundo de las Ciencias. Vol. 3 núm., 1, enero, ISSN: 2588-0748, 2018, pp. 653-669

DOI: $10.26820 /$ reciamuc/3.(1).enero.2019.653-669

URL: http://reciamuc.com/index.php/RECIAMUC/article/view/252

Código UNESCO: 3205 Medicina Interna

Tipo de Investigación: Artículo de Revisión

Editorial Saberes del Conocimiento

Recibido: 10/12/2018

Aceptado: 20/01/2019

Publicado: 30/01/2019

Correspondencia: stefany.campuzano@gmail.com
a. Médico; Guayaquil, Ecuador; stefany.campuzano@gmail.com
b. Médico; Guayaquil, Ecuador; carlosbajana0@gmail.com
c. Médico; Guayaquil, Ecuador; c.b.castro.fd@hotmail.com
d. Médico; Guayaquil, Ecuador; eccmd.84@hotmail.com 


\section{VIH/SIDA: Pruebas y su Efectividad}

Vol. 3, núm. 1., (2019)

Stefany Gianella Campuzano Lupera; Carlos Alexander Bajaña Gómez; Elvia María Córdova Cedeño; Carlos Eduardo Baque Castro

\section{RESUMEN}

El VIH/SIDA es uno de los principales problemas de salud a nivel mundial. Los avances de la medicina moderna han permitido desarrollar tratamientos que pueden llegar a minimizar el VIH hasta niveles imperceptibles, previniendo la aparición de la enfermedad, para lo cual es fundamental el diagnóstico temprano. La presente investigación trata de las pruebas de VIH/SIDA, así como de su efectividad. Para su desarrollo se llevó a cabo una recopilación y revisión de material documental bibliográfico. Para la detección y diagnóstico del VIH/SIDA, en la actualidad, existen muchas pruebas, las cuales se dividen en dos grupos, las pruebas de detección, cuyo resultado positivo es la base del siguiente grupo, el cual se trata de las pruebas de control, diagnóstico o confirmación. Existen una serie de normativas y procedimientos nacionales e internacionales para la aplicación debida de estas pruebas, cuya observación garantiza la minimización de resultados erróneos. Se concluye que las pruebas actuales para la detección y diagnóstico de VIH/SIDA son altamente efectivas, aún más si se siguen los procedimientos adecuados para su aplicación y valoración, así como las recomendaciones de la OMS, ONUSIDA y otras organizaciones nacionales en cuanto al orden, número y repetición de estas pruebas.

Palabras Claves: Virus; Síndrome; Inmunodeficiencia; Pruebas; Efectividad. 
Vol. 3, núm. 1., (2019)

Stefany Gianella Campuzano Lupera; Carlos Alexander Bajaña Gómez; Elvia María Córdova

Cedeño; Carlos Eduardo Baque Castro

\begin{abstract}
HIV / AIDS is one of the main health problems worldwide. Advances in modern medicine have allowed the development of treatments that can minimize HIV to imperceptible levels, preventing the onset of the disease, for which early diagnosis is essential. The present investigation deals with the HIV / AIDS tests, as well as their effectiveness. For its development a compilation and review of bibliographic documentary material was carried out. For the detection and diagnosis of HIV / AIDS, currently, there are many tests, which are divided into two groups, screening tests, whose positive result is the basis of the next group, which is the control tests, diagnosis or confirmation. There is a series of national and international regulations and procedures for the proper application of these tests, whose observation guarantees the minimization of erroneous results. It is concluded that the current tests for the detection and diagnosis of HIV / AIDS are highly effective, even more so if the proper procedures for its application and assessment are followed, as well as the recommendations of the WHO, UNAIDS and other national organizations regarding the order, number and repetition of these tests.
\end{abstract}

Key Words: Virus; Syndrome; Immunodeficiency; Tests; Effectiveness. 


\section{VIH/SIDA: Pruebas y su Efectividad}

Vol. 3, núm. 1., (2019)

Stefany Gianella Campuzano Lupera; Carlos Alexander Bajaña Gómez; Elvia María Córdova Cedeño; Carlos Eduardo Baque Castro

\section{Introducción.}

El VIH (virus de inmunodeficiencia humana es el virus causante del SIDA (síndrome de inmunodeficiencia adquirida). "Cuando una persona se infecta con VIH, el virus ataca y debilita al sistema inmunitario. A medida que el sistema inmunitario se debilita, la persona está en riesgo de contraer infecciones y cánceres que pueden ser mortales", en cuyo caso la enfermedad se llama SIDA. Es un virus que una vez que se transmite a un cuerpo habita en él de por vida. (Biblioteca Nacional de Medicina de los Estados Unidos, 2018)

Este virus es uno de los mayores problemas para la salud pública en todo el mundo superando hasta la fecha los 35 millones de vidas. En el 2.016, se estima que fallecieron un millón de personas a nivel mundial por causas relacionadas con este virus. Asimismo, para finales de ese mismo año existía un aproximado de 36,7 millones de personas infectadas por el $\mathrm{VIH}$ en todo el mundo, con un total de 1,8 millones de nuevas infecciones. "El $54 \%$ de los adultos y el $43 \%$ de los niños infectados están en tratamiento antirretrovírico (TAR) de por vida”. (Organización Mundial de la Salud, 2018)

El VIH a pesar de ser una enfermedad de por vida, gracias a los innumerables avances de la ciencia, hoy en día, puede tratarse efectivamente y dicha efectividad depende básicamente del diagnóstico temprano, es allí donde radica la base del éxito, en la prueba para diagnosticar este virus.

ONUSIDA, (2018) organización pionera en el mundo en la lucha contra el VIH/SIDA destaca que la única manera de estar seguro de que una persona tienes VIH es por medio de una prueba. Toda persona que crea haber estado en riesgo de contraer el virus, debe practicarse la 
Vol. 3, núm. 1., (2019)

Stefany Gianella Campuzano Lupera; Carlos Alexander Bajaña Gómez; Elvia María Córdova Cedeño; Carlos Eduardo Baque Castro

prueba con la finalidad de diagnosticar el virus lo antes posible y comenzar con un tratamiento temprano en caso de que el resultado resulte positivo. Es fundamental la administración del tratamiento temprano, ya que mientras más pronto se inicie el tratamiento, más posibilidades de estar sano y menos posibilidades de transmitir el virus tendrá la persona. (p. 3)

"La falta de diagnóstico del VIH representa un obstáculo importante para poner en práctica la recomendación de la OMS que preconiza ofrecer tratamiento antirretrovírico (TAR) a todas las personas infectadas por el VIH”. En la actualidad, más de 18 millones de personas con el virus reciben TAR y un número similar no tiene acceso al tratamiento, además de que la mayoría de estas desconoce su estado serológico. Igualmente, el $40 \%$ de las personas que viven con el virus, lo cual representa unas 14 millones de personas, no conocen su condición con respecto del virus. (Organización Mundial de la Salud, 2016)

El hecho anterior es confirmado por ONUSIDA, (2016) quienes han destacado en muchas oportunidades que pese al avance en la promoción del diagnóstico del VIH, aún la mitad de todas las personas que viven con el virus desconocen su estado, lo cual es alarmante y exige mayores esfuerzos para cerrar la brecha de la prueba. La mayor barrera para ampliar el tratamiento del virus en la actualidad está constituido por el diagnóstico tardío de la infección por el virus. (p. 14)

En este sentido, Las Naciones Unidas se han fijado metas, entre estas una de las principales es diagnosticar el $90 \%$ de las infecciones por el VIH para el año 2020. Se estima que en la actualidad solo el 70\% de las personas con VIH saben de su condición. (ONUSIDA, 2017) 


\section{VIH/SIDA: Pruebas y su Efectividad}

Vol. 3, núm. 1., (2019)

Stefany Gianella Campuzano Lupera; Carlos Alexander Bajaña Gómez; Elvia María Córdova Cedeño; Carlos Eduardo Baque Castro

Los esfuerzos de organizaciones como la OMS, ONUSIDA y el esfuerzo mancomunado de los países en el mundo han incluir en sus políticas de estado todo lo referencte en la lucha contra el VIH/SIDA, han dado una respuesta positiva en la eliminación de la transmisión del virus. Tal es el caso de Cuba, según la Biblioteca Médica Nacional de la República de Cuba, (2018) para el año 2.015 este fue el primer país, a nivel mundial, en recibir "la validación de la eliminación de la transmisión materno-infantil del VIH y la sífilis congénita por parte de la OMS”. Sus esfuerzos estuvieron orientados en asegurar el acceso temprano a la atención prenatal y a las pruebas para detectar el VIH y la sífilis, por ende, promovieron un tratamiento temprano tanto para las mujeres con diagnóstico positivo, como para sus bebés. (p. 2, 3)

Con base a la revisión de material actualizado y fidedigno, la presente investigación está orientada a plasmar la información relativa a las pruebas para el diagnóstico del VIH, dada la importancia para el tratamiento, control y eliminación de la enfermedad. Asimismo, los aspectos relacionados con la efectividad de las pruebas y algunas consideraciones para obtener diagnósticos más precisos.

\section{Materiales y Métodos.}

El presente estudio se realizó mediante una recopilación y revisión de material documental bibliográfico que se utilizó para documentar y plasmar lo relacionado con las pruebas de VIH/SIDA así como la efectividad de dichas pruebas.

Respecto a la metodología aplicada, para Arias, (2012) "la investigación documental es un proceso basado en la búsqueda, recuperación, análisis, crítica e interpretación de datos 
Vol. 3, núm. 1., (2019)

Stefany Gianella Campuzano Lupera; Carlos Alexander Bajaña Gómez; Elvia María Córdova Cedeño; Carlos Eduardo Baque Castro

secundarios, es decir, los obtenidos y registrados por otros investigadores en fuentes documentales: impresas, audiovisuales o electrónicas". (p. 27).

En razón de lo anterior, la presente investigación se enmarca en la clasificación de investigación de tipo documental por el proceso usado para su elaboración.

\section{Resultados.}

Detección y diagnóstico del VIH

Detectar el VIH es un proceso de dos pasos, que incluye un examen de detección y pruebas de control. Estos exámenes se pueden realizar con sangre extraída de una vena, una muestra de sangre obtenida pinchando un dedo, un hisopo con una muestra de saliva o una muestra de orina. Los exámenes de detección verifican la infección con VIH, mientras que los de seguimiento también conocidos como pruebas confirmatorias, se realizan en los casos en que la prueba de detección ha resultado positiva. (Biblioteca Nacional de Medicina de los Estados Unidos, 2017)

Existen muchos tipos de pruebas de detección, los más modernos ofrecen resultados hasta en 20 minutos y pueden ser practicados en el domicilio, lo cual garantiza una mayor confidencialidad. Por otra parte, las pruebas de control, seguimiento o pruebas de confirmación, por lo general, se llevan a cabo cuando la prueba de detección ha obtenido un resultado positivo.

En este orden de ideas generales, tenemos que hacen falta dos exámenes o más para diagnosticar con precisión el VIH. 


\section{VIH/SIDA: Pruebas y su Efectividad}

Vol. 3, núm. 1., (2019)

Stefany Gianella Campuzano Lupera; Carlos Alexander Bajaña Gómez; Elvia María Córdova Cedeño; Carlos Eduardo Baque Castro

Igualmente, es importante destacar que las personas con VIH no tienen SIDA, si una persona es detectada con VIH puede tomar tratamiento para prevenir la aparición del SIDA, es tal el avance tecnológico, que si se inicia un tratamiento temprano este puede reducir el virus a niveles indetectables, de allí la importancia de las pruebas.

Dentro de las pruebas de detección encontramos tres tipos para diagnosticar la infección por el VIH: las pruebas de anticuerpos las cuales examinan si hay anticuerpos contra el VIH en la sangre o en secreciones bucales. Estas son la mayoría de las pruebas rápidas y domiciliarias. Las pruebas de antígenos y anticuerpos las cuales detectan antígenos (una parte del virus), además de anticuerpos contra el VIH. Y las pruebas de ácido nucleico (NAT) las cuales examinan la presencia del VIH en la sangre. (Departamento de Salud y Servicios Humanos de EE. UU., 2018)

Existe otra clasificación de las pruebas de detección, según Álvarez-Carrasco, (2017) las pruebas de diagnósticos indirectos que sólo muestran la respuesta inmunitaria (pruebas rápidas Ac, ELISA de tercera generación, IFI y WB) y las de diagnósticos directos que muestran la presencia del virus o sus constituyentes (pruebas rápidas Ag/Ac, antigenemia p24 y ELISA de cuarta generación). (p. 310)

\section{Pruebas de VIH en niños}

El VIH avanza con mayor rapidez en los niños, por lo tanto, las pruebas de carga viral son de gran importancia en los neonatos. Se estima que la mortalidad máxima en los niños que nacen con VIH está entre los dos y los tres meses de vida. Es importante destacar, que las pruebas de diagnóstico rápido son ineficaces hasta los 18 meses de edad, es por ello que la única 
Vol. 3, núm. 1., (2019)

Stefany Gianella Campuzano Lupera; Carlos Alexander Bajaña Gómez; Elvia María Córdova Cedeño; Carlos Eduardo Baque Castro

prueba del VIH viable en niños menores a esta referencia es una prueba virológica que se debe realizar entre las cuatro y las seis semanas de vida. (ONUSIDA, 2018)

\section{Alere $^{\text {TM }}$ q HIV-1/2 Detect (fabricado por Alere Technologies GmbH) y Xpert ${ }^{\circledR}$ HIV-1}

Qual Assay (fabricado por Cepheid AB).

No obstante, existen innovadoras tecnologías para el diagnóstico temprano del VIH en los bebés. Para el 2016, estas pruebas (Alere ${ }^{\mathrm{TM}}$ q HIV-1/2 Detect y Xpert® HIV-1 Qual Assay) recibieron la precalificación de la OMS. La prueba Xpert® se puede utilizar para detectar diversas enfermedades, requiere una fuente de alimentación continua, poca formación o mantenimiento y se usa con muestras de sangre, o bien, con pruebas con sangre seca en papel. Por otra parte, la plataforma Alere funciona hasta 8 horas con una batería, esto permite su uso en zonas remotas y rurales, en donde no existen infraestructuras de laboratorio ni profesionales sanitarios cualificados. (ONUSIDA, 2016)

Pruebas de detección de VIH

ELISA

ELISA es el acrónimo en inglés para enzimoinmunoanálisis de adsorción. También llamado Inmunoanálisis ligado a enzimas y Enzimoinmunoanálisis (EIA). Es una prueba de laboratorio usada para detectar anticuerpos en la sangre. Se realiza a través de una muestra de sangre. Esta muestra se envía a un laboratorio. Se utiliza para verificar la exposición al VIH. (Biblioteca Nacional de Medicina de los Estados Unidos, 2018) 


\section{VIH/SIDA: Pruebas y su Efectividad}

Vol. 3, núm. 1., (2019)

Stefany Gianella Campuzano Lupera; Carlos Alexander Bajaña Gómez; Elvia María Córdova Cedeño; Carlos Eduardo Baque Castro

En los casos en que una prueba rápida resulta positiva, realizar un ELISA es la recomendación, si esta también resulta reactiva se solicitará otra muestra sanguínea para realizar una segunda prueba de ELISA, esta vez por duplicado. Si está o ambas resultaran reactivas, en ese caso el diagnóstico presuntivo es de infección por el VIH. (Álvarez-Carrasco, 2017, p. 312)

\section{Prueba de carga viral}

Es una prueba poco usada por su alto costo. También llamadas prueba de ácido nucleico, NAT (siglas en inglés), prueba de amplificación de ácido nucleico, NAAT (siglas en inglés), PCR de VIH, prueba de ARN, cuantificación de VIH. Esta prueba mide la cantidad de VIH que hay en la sangre. Es costosa y se usa para resultados rápidos. Incluso a una persona diagnosticada con el virus se le puede practicar esta prueba para estudiar los efectos de los medicamentos en estos pacientes. Esta puede detectar el virus en la sangre a los pocos días de haberse infectado, con respecto a otras pruebas que pueden tardar semanas o meses para detectar una infección. La ventaja sobre las otras pruebas es la velocidad con la que se obtienen los resultados lo cual permite tanto iniciar el tratamiento como tomar las medidas necesarias para evitar la trasmisión del virus. (Biblioteca Nacional de Medicina de los Estados Unidos, 2018)

\section{Antigenemia $p 24$}

Se considera una prueba altamente específica, no obstante, su sensibilidad no es óptima, lo que la hace presentar falsos negativos, lo que limita su uso al diagnóstico precoz, sobre todo durante el periodo de ventana o cuando existe presunción de exposición al virus. Es por esta razón que se ha preferido incorporarla a los ELISA de cuarta generación. Esta prueba puede detectarse de once a trece días después de la infección, sus niveles de concentración permanecen 
Vol. 3, núm. 1., (2019)

Stefany Gianella Campuzano Lupera; Carlos Alexander Bajaña Gómez; Elvia María Córdova Cedeño; Carlos Eduardo Baque Castro

altos y detectables en sangre por un mes y medio aproximadamente, después de la infección. Durante el proceso, a medida que aparecen los anticuerpos anti-VIH, su concentración disminuye, aumentando los complejos p24/anti-p24. Resultados dudosos o débilmente positivos de esta prueba deben ser confirmados a través de la prueba de neutralización. (Álvarez-Carrasco, 2017, p. 314)

Pruebas de diagnóstico de VIH

\section{Recuento de linfocitos CD4}

También llamada recuento de linfocitos CD4, recuento de $\mathrm{CD} 4+$, recuento de $\mathrm{T} 4$, recuento de linfocitos $\mathrm{T}$ cooperadores, porcentaje de $\mathrm{CD} 4 \mathrm{y}$ conteo de células $\mathrm{T}$, es una prueba usada para medir la cantidad de linfocitos CD4 en sangre. Los linfocitos CD4 o linfocitos T4, "son glóbulos blancos que combaten infecciones y desempeñan un papel importante en el sistema inmunitario. El conteo de CD4 se usa para vigilar la salud del sistema inmunitario en personas infectadas con el VIH (virus de la inmunodeficiencia humana)". El VIH ataca y destruye los linfocitos CD4, dependiendo de la pérdida de linfocitos CD4, el sistema inmunitario tendrá dificultad para combatir infecciones, por lo tanto, permite tomar las medidas necesarias para reducir el riesgo de complicaciones graves por el VIH. Asimismo, los resultados de la prueba permiten medir la efectividad de los tratamientos contra el virus. (Biblioteca Nacional de Medicina de los Estados Unidos, 2018)

Las personas con SIDA periódicamente deben realizarse análisis de sangre con la finalidad de verificar su conteo de células CD4, a medida que el VIH daña el sistema 


\section{VIH/SIDA: Pruebas y su Efectividad}

Vol. 3, núm. 1., (2019)

Stefany Gianella Campuzano Lupera; Carlos Alexander Bajaña Gómez; Elvia María Córdova Cedeño; Carlos Eduardo Baque Castro

inmunitario, el conteo de CD4 disminuye. (Biblioteca Nacional de Medicina de los Estados Unidos, 2018)

\section{Inmunofluorescencia indirecta}

Tiene una sensibilidad y especificidad parecida a la prueba Western Blot (WB), inclusive su positividad puede ocurrir antes que el Western Blot, además es mucho más barata, hasta 11 veces menor, en cuanto al tiempo de ejecución se puede mencionar que también es menor, así como también posee una técnica más simple, es por estas razones que ha desplazado al WB. Su resultado positivo constituye el diagnóstico definitivo de la infección por el VIH y su resultado negativo, es definitiva, de no infección. (Álvarez-Carrasco, 2017, p. 314)

\section{Western Blot}

Es una prueba altamente específica pero por su alto costo se emplea básicamente para corroborar los resultados indeterminados de la IFI. Sus criterios de interpretación no se han unificado entre la OMS, el Centers for Disease Control and Prevention (CDC) norteamericano y la Cruz Roja norteamericana. En el Perú se utilizan los valores del CDC, que considera positiva la prueba de WB cuando aparecen la p24 + (gp160, gp120 o gp41) o la p41 + (gp160 o gp120). El resultado positivo confirma definitivamente la infección por el VIH; el negativo la descarta, excepto cuando existe evidencia de exposición reciente y reiterada a esta infección. En tales circunstancias se debe repetir el ensayo luego de tres y seis meses respectivamente. Cuando el resultado es indeterminado, que ocurre cuando sólo aparecen algunas bandas que no cumplen con los criterios del CDC, se recomienda repetir la prueba después de tres y seis meses, 
Vol. 3, núm. 1., (2019)

Stefany Gianella Campuzano Lupera; Carlos Alexander Bajaña Gómez; Elvia María Córdova Cedeño; Carlos Eduardo Baque Castro

dependiendo de los factores de riesgo identificables en cada caso. (Álvarez-Carrasco, 2017, p. 314)

Efectividad de las pruebas

Se pueden minimizar los riesgos de diagnósticos del VIH erróneos, mediante el uso de kits y procedimientos que cumplan con los requisitos de calidad de la OMS, así como, de otros requisitos nacionales o regionales que se deriven de este. "Se revisará la idoneidad de los procedimientos para la realización de pruebas... también se llevarán a cabo controles de calidad externos de manera periódica para detectar las causas potenciales de los diagnósticos erróneos de VIH”. (ONUSIDA, 2017)

"La OMS y ONUSIDA sostienen que a nadie se le debe diagnosticar como seropositivo en base a una única prueba del VIH que resulte positiva". Es imperioso tener claro que para la confirmación de un resultado positivo deben seguirse los procedimientos nacionales para las pruebas del VIH, inclusive, previo inicio de un tratamiento antirretrovírico permanente deben ser repetidas las pruebas. (ONUSIDA, 2017)

Álvarez-Carrasco, (2017) se refiere a las limitantes que presentan las pruebas diagnósticas que se expresan por medio de los falsos positivos (falsos reactivos) los cuales son aquellos casos en los que un individuo que no está infectado por VIH tiene pruebas diagnósticas positivas o reactivas. Estos son más frecuentes en las pruebas rápidas e infrecuentes en las confirmatorias. Y los falsos negativos (falsos no reactivos) son aquellos casos en los que el individuo infectado con VIH tiene pruebas diagnósticas negativas o no reactivas, lo cual es de baja ocurrencia dada la alta sensibilidad de las actuales pruebas de tamizaje. "La OMS 


\section{VIH/SIDA: Pruebas y su Efectividad}

Vol. 3, núm. 1., (2019)

Stefany Gianella Campuzano Lupera; Carlos Alexander Bajaña Gómez; Elvia María Córdova Cedeño; Carlos Eduardo Baque Castro

recomienda el empleo de dos pruebas de ELISA de diferente mecanismo de acción como estrategia alternativa para corroborar la infección por el VIH, pues si ambas resultaran reactivas, ello sería equivalente a una prueba confirmatoria”. (p. 313, 314)

Por otra parte, las pruebas rápidas para detectar VIH son efectivas en más de un 98\%, tomando en consideración que estas pruebas no son diagnósticas por lo que siempre hay que corroborar resultados por medio de una prueba confirmatoria. Es recomendable en estas pruebas, sobre todo en personas que han tenido prácticas de riesgos que las pueden llevar a adquirir VIH, dejar transcurrir tres meses para realizarse una prueba rápida, por cuanto existe un periodo llamado "de ventana", en donde no existen los niveles de anticuerpos necesarios contra el VIH, por lo que podrían resultar un falso negativo, es decir, salir los resultados negativos sin serlo. "Las personas que hayan tenido prácticas de riesgo y quieran tener resultados tienen que hacerse una prueba de ELISA (inmunoensayo) de cuarta generación, y los resultados se obtienen en 24 horas”. (Universidad de Guadalajara, 2015)

\section{Conclusiones.}

Existen muchas pruebas de detección y diagnóstico para el VIH y SIDA. La tecnología actual pone al alcance de la población mundial innovaciones que mejoran la exactitud, costo y menor tiempo en los resultados. Actualmente la OMS, ONUSIDA y otras organizaciones y países del mundo se han planteado metas para erradicar el VIH y el SIDA del mundo, uno de los objetivos para lograr esta meta es aumentar en un 90\% la aplicación de las pruebas de VIH, derrumbando limitantes como el acceso a la prueba, el costo, las leyes y la confidencialidad en torno a estos examenes y sus resultados. 
Vol. 3, núm. 1., (2019)

Stefany Gianella Campuzano Lupera; Carlos Alexander Bajaña Gómez; Elvia María Córdova Cedeño; Carlos Eduardo Baque Castro

La importancia de las pruebas de VIH radica en que mientras más pronto sea diagnosticado el virus mayor será la oportunidad de tratarla, evitar la aparición del SIDA y sus consecuencias mortales.

En cuanto a la efectividad de las pruebas, se debe tener en cuenta que existen pruebas de detección, cuyo resultado positivo debe ser respaldado por una prueba confirmatoria y la repetición de la misma inclusive, con la práctica de varias pruebas, aunado a los avances tecnológicos, el grado de confiabilidad en el resultado es muy alto.

Actualmente en el mundo existen muchas polémicas en cuanto a la efectividad de una prueba u otra, muchas veces por razones políticas o por el desconocimiento general del tema, no obstante, las pruebas son efectivas si se siguen los procedimientos regulados para cada una de ellas y se consulta un especialista de salud que supervise el proceso para evitar los falsos positivos o negativos.

La información que puedan manejar las personas y romper con los paradigmas de rechazo es fundamental para una mayor práctica de las pruebas. La detección a tiempo del VIH puede ser sinónimo de que los individuos puedan llevar vidas largas y plenas. Gracias a la tecnología actual los tratamientos tempranos pueden lograr una carga vírica baja o indetectable, lo cual significa que el tratamiento ha dado resultado y que el VIH se ha suprimido.

\section{Bibliografía.}

Álvarez-Carrasco, R. I. (11 de Octubre de 2017). Interpretación de las pruebas usadas para diagnosticar la infección por virus de la inmunodeficiencia humana. Acta Médica Peruana, 34(04), 309-316. Recuperado el 12 de Mayo de 2019, de http://www.scielo.org.pe/pdf/amp/v34n4/a09v34n4.pdf 


\section{VIH/SIDA: Pruebas y su Efectividad}

Vol. 3, núm. 1., (2019)

Stefany Gianella Campuzano Lupera; Carlos Alexander Bajaña Gómez; Elvia María Córdova Cedeño; Carlos Eduardo Baque Castro

Arias, F. (2012). El Proyecto de Investigación. Introducción a la metodología científica. (6ta ed.). Caracas, Venezuela: Episteme. Recuperado el 12 de mayo de 2019, de https://ebevidencia.com/wp-content/uploads/2014/12/EL-PROYECTO-DE-

INVESTIGACI\%C3\%93N-6ta-Ed.-FIDIAS-G.-ARIAS.pdf

Biblioteca Médica Nacional de la República de Cuba. (febrero de 2018). VIH/Sida Estadísticas Mundiales. Factográfico de Salud, 4(2), 1-13. Recuperado el 14 de mayo de 2019, de http:/files.sld.cu/bmn/files/2018/02/factografico-de-salud-febrero-2018.pdf

Biblioteca Nacional de Medicina de los Estados Unidos. (18 de Mayo de 2017). MedlinePlus. $\begin{array}{llllll}\text { Recuperado el } & 14 & \text { de } & \text { Mayo } & \text { de }\end{array}$ https://medlineplus.gov/spanish/ency/article/003538.htm

Biblioteca Nacional de Medicina de los Estados Unidos. (05 de Junio de 2018). MedlinePlus. $\begin{array}{llllll}\text { Recuperado el } & 15 & \text { de } & \text { Mayo } & \text { de } & \text { de }\end{array}$ https://medlineplus.gov/spanish/ency/article/000594.htm

Biblioteca Nacional de Medicina de los Estados Unidos. (08 de Octubre de 2018). MedlinePlus. $\begin{array}{lllllll}\text { Recuperado el } & 10 & \text { de } & \text { Mayo } & \text { de }\end{array}$ https://medlineplus.gov/spanish/ency/article/003332.htm

Biblioteca Nacional de Medicina de los Estados Unidos. (07 de Noviembre de 2018). MedlinePlus. Recuperado el 14 de Mayo de 2019, de https://medlineplus.gov/spanish/pruebas-de-laboratorio/prueba-de-carga-viral/

Biblioteca Nacional de Medicina de los Estados Unidos. (07 de Noviembre de 2018). MedlinePlus. Recuperado el 16 de Mayo de 2019, de https://medlineplus.gov/spanish/pruebas-de-laboratorio/recuento-de-linfocitos-cd4/

Departamento de Salud y Servicios Humanos de EE. UU. (19 de Noviembre de 2018). InfoSIDA. Recuperado el 09 de Mayo de 2019, de https://infosida.nih.gov/understanding-hivaids/fact-sheets/19/47/pruebas-de-deteccion-del-vih

ONUSIDA. (2016). unaids.org. Recuperado el 14 de mayo de 2019, de http://www.unaids.org/sites/default/files/media_asset/UNAIDS-strategy-20162021_es.pdf

ONUSIDA. (23 de Junio de 2016). unaids.org. Recuperado el 14 de Mayo de 2019, de http://www.unaids.org/es/resources/presscentre/featurestories/2016/june/20160623_HIVdetection-infants

ONUSIDA. (29 de aGOSTO de 2017). unaids.org. Recuperado el 14 de Mayo de 2019, de http://www.unaids.org/es/resources/presscentre/featurestories/2017/august/20170829_HI $\mathrm{V}$-testing-services 
Vol. 3, núm. 1., (2019)

Stefany Gianella Campuzano Lupera; Carlos Alexander Bajaña Gómez; Elvia María Córdova Cedeño; Carlos Eduardo Baque Castro

ONUSIDA. (01 de Diciembre de 2018). unaids.org. Recuperado el 14 de Mayo de 2019, de http://www.unaids.org/sites/default/files/media_asset/what-you-need-to-know-about-hivtesting_es.pdf

ONUSIDA. (22 de Noviembre de 2018). unaids.org. Recuperado el 15 de Mayo de 2019, de http://www.unaids.org/es/resources/presscentre/pressreleaseandstatementarchive/2018/no vember/20181122_WADreport_PR

Organización Mundial de la Salud. (29 de noviembre de 2016). who.int. Recuperado el 14 de mayo de 2019, de https://www.who.int/es/news-room/detail/29-11-2016-who-issuesnew-guidance-on-hiv-self-testing-ahead-of-world-aids-day

Organización Mundial de la Salud. (19 de julio de 2018). who.int. Recuperado el 13 de mayo de 2019, de https://www.who.int/es/news-room/fact-sheets/detail/hiv-aids

Universidad de Guadalajara. (04 de Mayo de 2015). Universidad de Guadalajara. (M. E. Loera, Editor) Recuperado el 14 de Mayo de 2019, de http://www.udg.mx/es/noticia/pruebarapida-para-detectar-vih-tiene-una-efectividad-de-hasta-98 